\title{
Experience in Developing an Astronomy Program in Paraguay
}

\author{
Alexis E. Troche-Boggino \\ Observatorio Astronomico "Alexis Emilio Troche Boggino", Universidad \\ Nacional de Asunción, Facultad Politécnica, 01 Agencia Postal Campus \\ U.N.A., Central XI, Paraguay. e-mail: atroche@pol.com.py
}

\begin{abstract}
Encouraging IAU-ISYA schools were held in Argentina (1974) and Brazil (1977 and 1995). Contacts with IAU Commission 46 were soon established and the author became the responsible person for setting up a supporting framework for astronomy in Paraguay. A proposal for an IAU-VLP program was made and the program began in 1988, running until 1994. The IAU Traveling Telescope and another telescope were borrowed for hands-on astronomy. Three students went to the Brazil ISYA in 1995 and two others began to work as astronomers abroad. Others became high-school and university instructors. A small library was opened, thanks to donations from IAU members. The need to find jobs for these young astronomers led to the further development of an Astronomy Center around the National Astronomical Observatroy.
\end{abstract}

\section{Introduction}

Please, do not confuse Paraguay with its neighbor country Uruguay. Paraguay is a land-locked country between Argentina, Brazil and Bolivia. Its population is over five million inhabitants and its capital city is Asunción (latitude $-25^{\circ} 18^{\prime}$, longitude $57^{\circ} 35^{\prime} \mathrm{W}$ ).

I became active in astronomy education 26 years ago. There was little astronomy taught in my country at that time, but I found opportunities for training high-school teachers. A new program for secondary schools was in a trial stage at that time. Basic astronomy topics were requested as a part of three high-school courses in natural science and as a part of the course in mathematics for the last year. Also, an elective astronomy course for junior students of physics were on schedule at National University of Asunción.

So, I used such opportunities to practice astronomy teaching as a university instructor and later I started to train high-school teachers in topics related to our science. I still do that and I have some former students in the same job at the Instituto Superior de Educación of the Ministry of Education and Culture.

Encouraging IAU-ISYA (International Schools for Young Astronomers) were held in Argentina in 1974 and later ones in Brazil in 1977 and 1995. Contacts 
with IAU Commission 46 were soon established and I learned what to do to start an astronomical supporting framework for my country.

Let me quote a motto from Dr. Cecylia Iwaniszewska, to whom I am grateful for continous kind assistance. "In order to reach each new generation with basic astronomy, we need to show its memebers the most outstanding facts, as well as to build a supporting frame".

What is the supporting frame's relevance? First, it means good reading materials, practical work, well-trained teachers, an astronomical observatory, a planetarium etc.

Second, with a poor framework it may happen that students may learn poorly and come to believe that they are experts. This means, in turn, instruction will become sketchy and few real experts will get good jobs. In my experience, one of the greatest difficulties in developing countries is that many people, both in government and universities, get their jobs not by their skill and knowledge, but through politics and personal connections. So many of our best students go abroad.

There are problems with the English language, which is less well-known the farther a country is from North America. This is bad for our students who cannot read some excellent astronomy pages on the Internet. What advice can you give to overcome this language barrier?

Among important visitors from the IAU astronomers were J. Sahade and R.M. West. These visits led to a Visiting Lecturers Program (VLP) for Paraguay, which began in 1988 and ran until 1994. I worked as local coordinator of six excellent astronomical courses with astronomers from Argentina, México and Italy. In particular, D.G. Wentzel's readiness to help, providing supporting material and visiting, improved the VLP.

Among the results of the IAU-VLP, two students went abroad for further study and practical work in astronomy. Another two attended the ISYA in Brazil in 1995. Two former VLP students are doing their doctoral work in physics abroad. Other former students are university and high-school instructors. A small astronomy library was has been formed with the help of donations from Canadian, American and other astronomers.

The IAU Traveling Telescope was lent for the last two courses in Astronomy. Mr. and Mrs. Eduardo Parini, local amateur astronomers, also lent us a 20-cm Celestron from their own private observatory. We consider the Parinis to be our "Maecenas" because of their generous assistance with their own equipment, maps etc. They acted as hosts, in their home, to many visiting professors and astronomers, and opened their observatory to students, amateurs and the public.

Natural events are of course useful. The visit of Halley's comet led to the formation of a first amateur astronomers' club in 1985 and two others were formed at the time of the total solar eclipse of November 3, 1994.

The president of one of these clubs, Dr Blas Severin founder of the Asociación de Amigos de la Astronomía, organizes regular star parties and gives lectures for beginning amateurs. Furthermore, he founded three amateur clubs at high schools.

A third group, the Sociedad de Estudios Astronómicos, publishes a bimonthly bulletin called Antares. Their members teach astronomy to the public and are active participants in international meetings. They are organizing a 


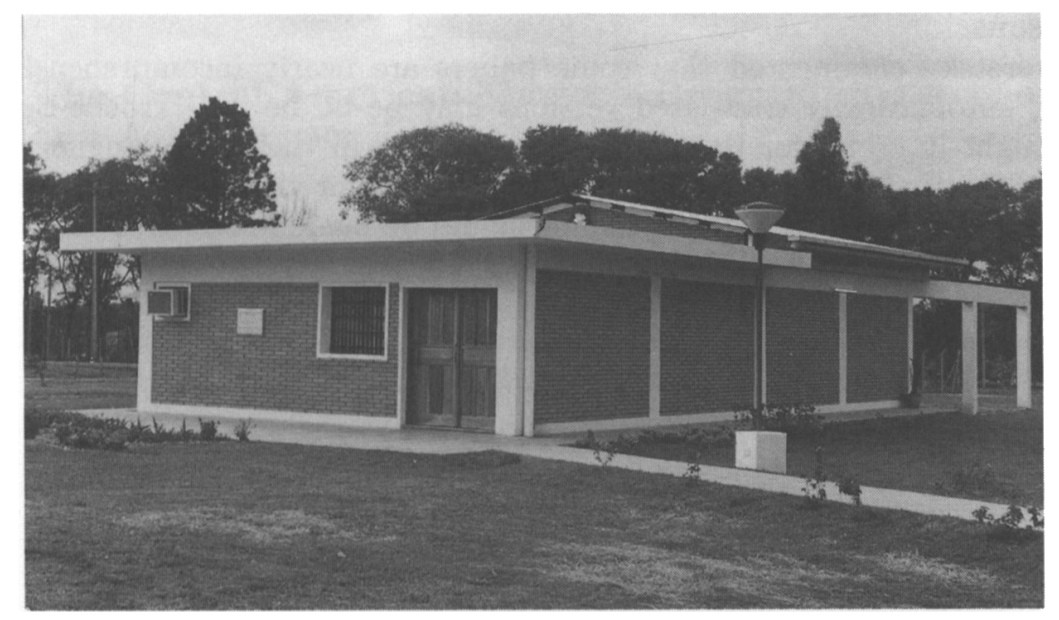

Figure 1. The Astronomical Observatory of the Universidad Nacional de Asunción. The Observatory was inaugurated on June 6th, 2000 and named after the author of this paper.

meeting for Latin-American amateur astronomers next year. Thus, amateur astronomers help to build a strong framework for beginning an astronomy program.

However, not many of our physics students get really involved in astronomy activities because of the lack of jobs and low salaries. We needed an "attractor" for them. Our project to obtain instruments for a professional astronomical observatory began to be realized last year with the donation of a 45-cm Cassegrain reflector, a photoelectric photometer of high accuracy and CCD cameras and image-processing equipment by the Japanese Government O.D.A., last year.

The Astronomical Observatory of Universidad Nacional de Asunción was inaugurated last June 6th, at the University's Polytecnic School. A young physicist, Mr. Fredy Doncel was trained in Japan for six months in the use of the instruments. He is doing well in his new job.

We are looking for a senior veteran astronomer from abroad, to help us to work with photometry of binary stars and research on variable stars. Further, we welcome astronomers from the IAU who would like to assist by visiting us and giving astronomical lectures. We are particularly grateful to Drs D.G. Wentzel, R. Vicente and M. Kitamura for the many lectures they gave us few years ago.

Dr. J. R. Percy visited us for about a week in 1997. He did a remarkable job presenting several lectures, which were very useful, particularly for our astronomy instructors. Further he suggested to us the development of an Astronomy Center in Paraguay and he gave a strong support to the project of an astronomical observatory.

We would like to have your advice and help for further development of this project. Please, remember our motto: "Let all students who wish to learn astronomy do so as far as they are able and let us give them the opportunity as much as possible". 


\section{Discussion}

Dworetsky commented that some papers are nearly incomprehensible in English; automatically translated versions may be no better! Troche-Boggino still thought it useful for people to have versions in their own languages. In reply to Rijsdijk he said that there were no charges for visiting the Observatory (on two days a week) but arrangements should be made in advance. 